\title{
Thermal Plasma Generators with Water Stabilized Arc
}

\author{
Milan Hrabovsky* \\ Institute of Plasma Physics ASCR, v.v.i., Praha, Czech Republic
}

\begin{abstract}
Electric arc stabilized by a vortex of liquid, mostly water, is used in a special type of plasma torch for generation of thermal plasma jet with extreme plasma parameters. The paper presents analysis of properties and processes of water stabilized arcs and discusses differences from common gas stabilized arcs. Plasma torch utilizing stabilization of arc column by combination of gas flow and liquid vortex is described and its properties are presented.
\end{abstract}

\section{INTRODUCTION}

Thermal plasmas are commonly generated in electric arcs that are stabilized by flowing gas or in inductively coupled discharges. Typically, inductively coupled plasma torches are characterized by plasma temperatures from $5000 \mathrm{~K}$ to 10 $000 \mathrm{~K}$ [1]. Averaged temperatures in torches with nontransferred electric arcs with gas stabilization are somewhat higher, usually in the range from $8000 \mathrm{~K}$ to $16000 \mathrm{~K}[1,2]$. Average plasma enthalpies, determined as a ratio of power of plasma to the flow rate of plasma forming gas, vary from 1 to $100 \mathrm{MJ} / \mathrm{kg}$. Further increase of plasma temperatures and enthalpies is limited, as flowing gas protects the arc chamber walls from thermal overloading and thus a minimum possible gas flow rate exists for given arc power. Higher thermal loading is possible if the walls are created by liquid and an arc is stabilized by wall evaporation. Thus, liquidstabilized arcs can be utilized as sources of thermal plasmas with extremely high temperatures and enthalpies. Besides apparent advantage of liquid-stabilized generators that no gas supply is needed, there are many other differences between gas and liquid generators in plasma processes, plasma properties and especially in performance characteristics in plasma processing.

Although water stabilized arc, sometimes called as Gerdien arc, has been known for a long time as a source of thermal plasma with very high temperature, exact investigation of its properties and processes was realized only in the last years. The arc with the stabilization of arc column by water vortex was first described more than eighty years ago by Gerdien and Lotz [3, 4]. Basic experimental investigations of water stabilized arcs were performed in the fifties. Maecker et al. [5, 6] measured electric characteristics of the arc, several investigators measured arc plasma temperatures using methods of emission spectroscopy $[7,8]$. All authors reported very high plasma temperatures in the arc column with maximum about $50000 \mathrm{~K}$ in the centerline position. The principle of arc stabilization by water vortex was utilized in the plasma torch designed for plasma spraying and cutting [9].

*Address correspondence to this author at the Institute of Plasma Physics ASCR, v.v.i., Praha, Czech Republic; E-mail: hrabovsky@ipp.cas.cz
In the last decade, water-stabilized arcs have been intensively investigated, basic processes in the arc column have been described and models of arc with water stabilization have been formulated. Complex experimental data about properties of the arc and parameters of generated plasma were presented in [10-13], two-dimensional numerical model of Gerdien arc was formulated in $[14,15]$.

Physical limits of the two principles, the gas and the liquid arc stabilization, do not allow generation of plasmas with parameters in a wide range between the two principles. Therefore new type of plasma torch, hybrid gas/water torch, was designed. The torch utilizes combination of principles of stabilization of arc by gas flow and water vortex, and provides thermal characteristics typical for water torches but with increased plasma flow rates and densities characteristic for gas torches.

This paper presents survey of arc and plasma parameters of liquid and hybrid torches and discusses physical mechanisms that control plasma properties.

\section{PROCESSES AND PROPERTIES OF GERDIEN ARC}

Schematic picture of plasma torch with Gerdien arc is shown in Fig. (1). The chamber is divided into several sections where water is injected tangentially and water vortex is created. The inner diameter of the vortex is determined by the diameter of the holes in the segments between the sections. Water is exhausted at two positions at the ends of the arc chamber. Consumable cathode is created by graphite rod, anode made of copper disc with internal cooling is located outside the arc chamber downstream of the exit nozzle. The anode disc rotates to reduce strong electrode erosion in steam plasma.

Evaporation from the inner wall of water vortex, surrounding the arc column, and heating and ionization of produced steam, are principal mechanisms that produce arc plasma. The evaporation is induced by the absorption of a fraction of Joule power dissipated within the conducting arc core. Steam inflow and its heating and ionization produce an overpressure within the arc chamber and steam plasma is accelerated towards the exit nozzle. The arc properties are thus controlled by processes influencing evaporation of the wall and by radial energy transport from the arc core to the walls. 


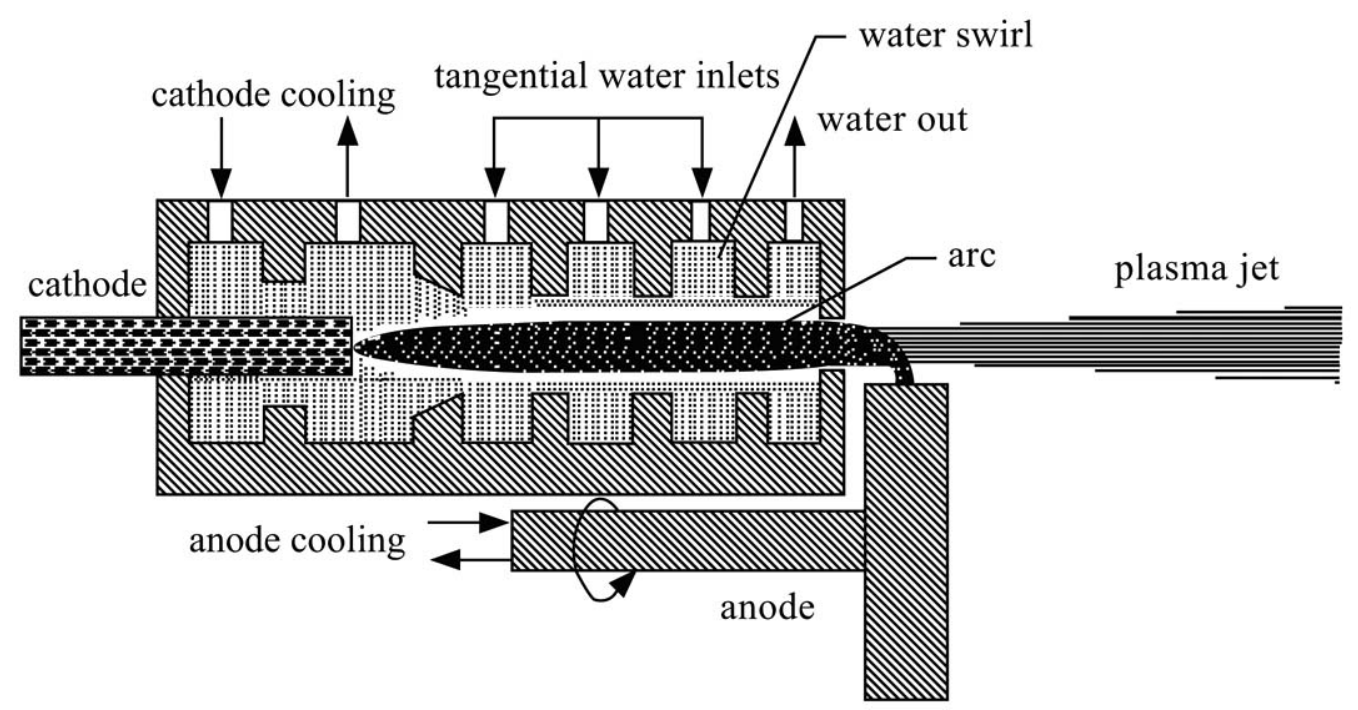

Fig. (1). Schematics of plasma torch with Gerdien arc.

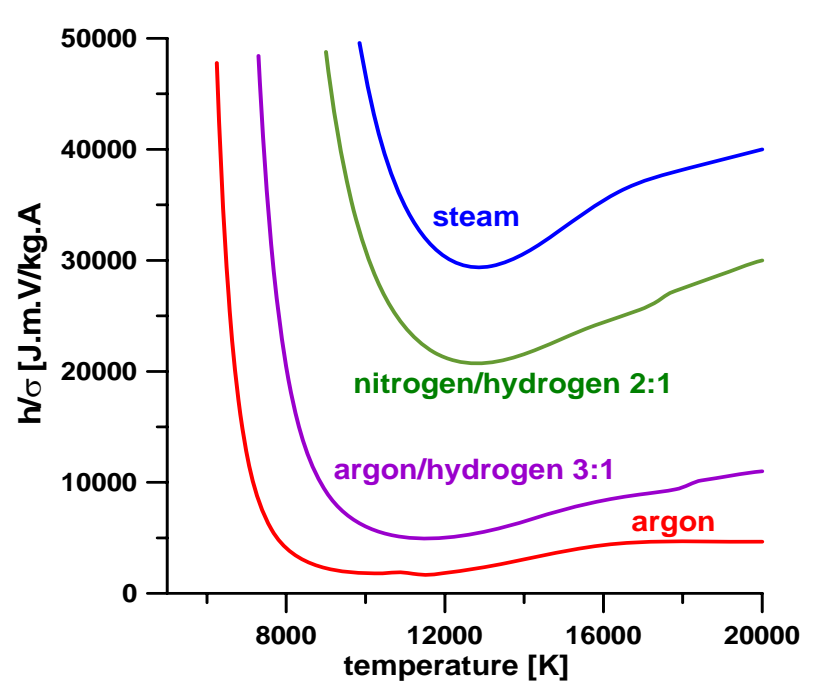

Fig. (2). Ratio of plasma enthalpy to electrical conductivity for several gases used in dc arc torches.

Specific characteristics of plasma torches with Gerdien arc follow from both the principle of arc stabilization and from material properties of steam plasma. Plasma produced by ionization of steam is characterized by high thermal conductivity, high enthalpy and high sound velocity $[16,17]$. In Fig. (2) the ratio of plasma enthalpy to electrical conductivity is shown for several gases used in plasma spraying torches. Arc voltage, and consequently arc power, is approximately proportional the square root of this ratio for given plasma mass flow rate. Therefore arc torches operated with steam are characterized by high arc power. High thermal conductivity of steam plasma is also important for some plasma processing technologies.

The main difference of Gerdien arc from more common gas stabilized torches follows from the fact that plasma forming gas, in case of Gerdien arc it is steam, flows transversally from walls surrounding the arc column, while in gas torches plasma gas enters the chamber at the cathode end and it is heated during its flow along the arc column. In case of Gerdien arc a sheath of cold steam separating arc plasma from water wall is created. Radial inflow of this steam causes a constriction of arc and an increase of plasma temperature, and reduces heat transfer to the wall. These processes result in reduced evaporation rate and thus plasma mass flow rate, and consequently in high plasma temperatures and enthalpies.

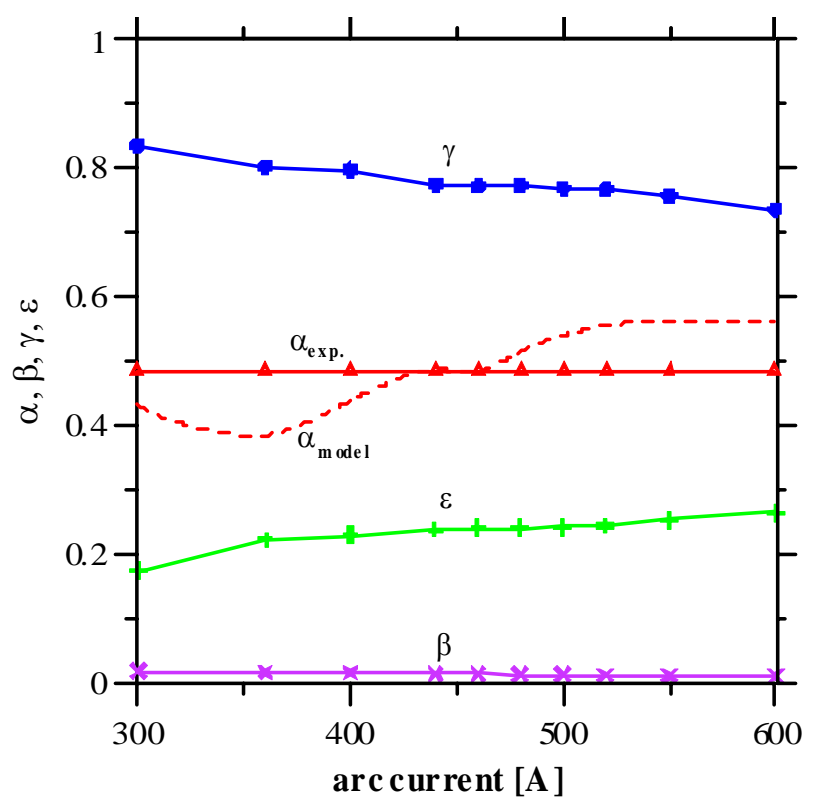

Fig. (3). Basic components of radial heat transfer in the column of water-stabilized arc. $\alpha$ - fraction of total arc power transferred radially, $\beta$ - fraction of radially transferred power spent for evaporation, $\varepsilon$ - fraction of radially transferred power absorbed in steam sheath, $\gamma$ - fraction of radially transferred power corresponding to power loss to water.

Energy balance of radial heat transfer, that determines formation of steam sheath, evaporation rate of water, and consequently plasma mass flow rate, is decisive for arc characteristics and plasma properties. Typically, very low fraction of total arc energy is spent for evaporation while absorption in vapor is relatively high. This is the main cause 
of low mass flow rate of plasma and very high plasma enthalpy and temperature that are typical for water torches. Fig. (3) presents typical balance of radial heat transfer in Gerdien arc, evaluated from the measurement of characteristics of arc in water stabilized torch [11]. It can be seen that about $50 \%$ of arc power is transferred radially $(\alpha)$, but only $2 \%$ of this radially transferred energy is spent for evaporation $(\beta)$ and more than $20 \%$ of this energy is spent for heating of evaporated steam $(\varepsilon)$. Very low fraction of total arc power spent for the production of plasma gas is the main cause of extremely high plasma temperatures and velocities accompanied by low mass flow rates through the exit nozzle.

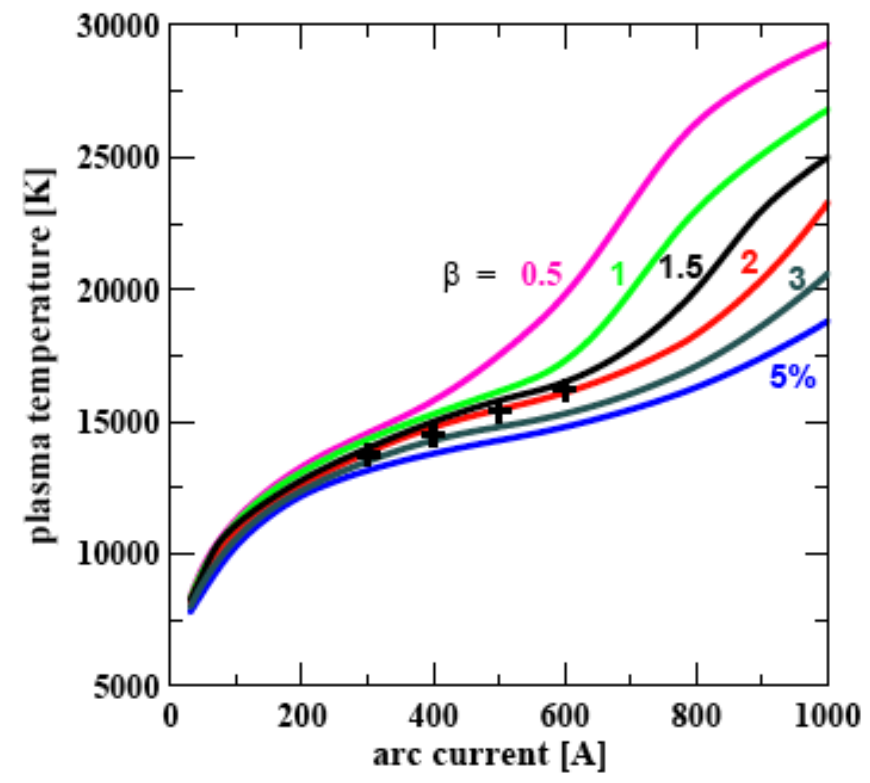

Fig. (4). Dependence of plasma temperature on arc current for several values of parameter $\beta$. Results of channel arc model [18]. Diameter of water vortex $3.5 \mathrm{~mm}$, length of arc column $55 \mathrm{~mm}$.

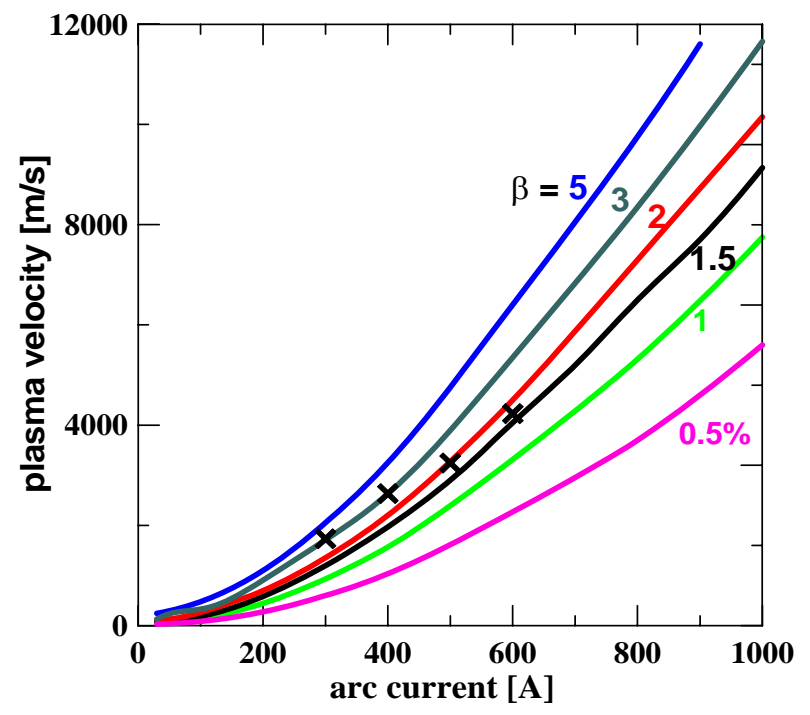

Fig. (5). Dependence of plasma velocity on arc current for several values of parameter $\beta$. Results of channel arc model [18]. Diameter of water vortex $3.5 \mathrm{~mm}$, length of arc column $55 \mathrm{~mm}$.
Effect of steam evaporation, which is principal mechanism controlling arc parameters, on plasma temperature and velocity is illustrated in Figs. $(4,5)$, where dependence of mean plasma temperature and velocity on fraction $\beta$ of radially transferred energy spent for evaporation is shown. The curves were calculated by solution of simple theoretical model [18] of Gerdien arc with input data corresponding to experimental water stabilized plasma torch WSP $160 \mathrm{~kW}$. The points correspond to measured values for this torch.

The results of measurements correspond to the curves calculated for values of parameter $\beta=1.5-2$, it means for fraction of radially transferred energy spent for evaporation equal to 1.5 . to $2 \%$. This value corresponds well to the value of $\beta$ evaluated from experiments in [11]. An increase of $\beta$, that can be achieved by an increase of absorption coefficient of liquid or by change of thermodynamic coefficients for evaporation by additives to water, results in substantial increase of plasma velocity and torch efficiency, while plasma temperature and electric field intensity are reduced. This may be important for application of the torch in plasma spraying, where increase of plasma velocity and torch efficiency may have positive effect on performance characteristics of the torch.

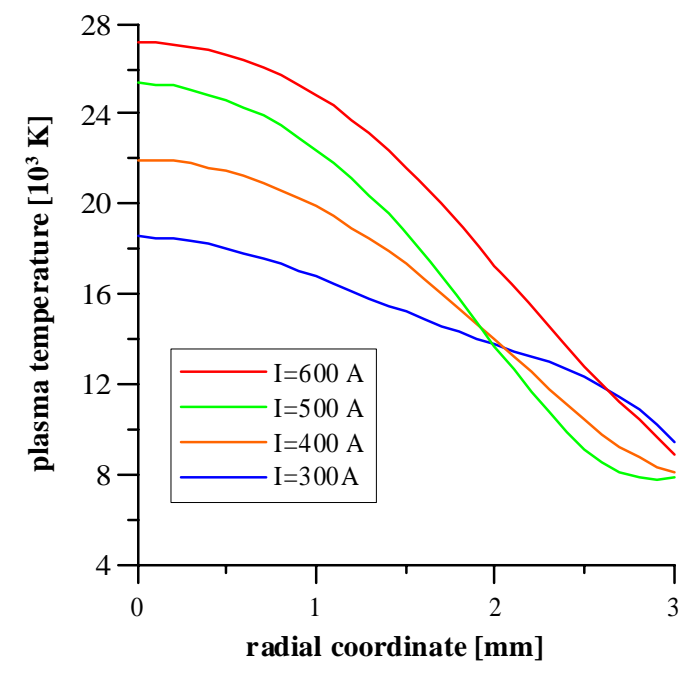

Fig. (6). Profiles of plasma temperature for several arc currents, measured on water stabilized plasma torch [11].

Fig. (6) presents measured profiles of plasma temperature determined by emission spectroscopy from analysis of $\mathrm{H}_{\beta}$ and oxygen ion lines [11]. Fig. (7) shows measured dependence of plasma velocity, determined experimentally from analysis of fluctuations of plasma radiation, on distance from the nozzle exit.

As walls of stabilizing cylinder are created by water, arc can be operated at substantially higher power in case of water torches. Fig. (8) presents comparison of operation regimes of water stabilized torches and conventional gas stabilized torches, characterized by levels of arc power and plasma mass flow rate. Low mass flow rates of plasma for 


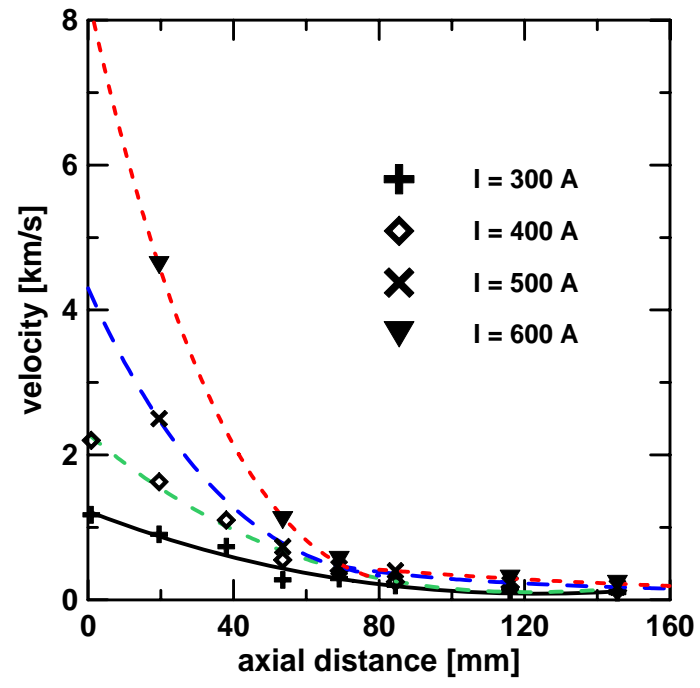

Fig. (7). Dependence of plasma velocity on distance from exit nozzle, measured on water stabilized plasma torch [11].

water torches follows from the energy balances of radial heat transfer. For gas torches mass flow rates can be controlled independently. However, lower limit of mass flow rate is given by necessity to protect walls of arc chamber by gas flow. It can be seen that water plasma torches are characterized by very low mass flow rates. This fact results in high plasma enthalpies. Typical values of mean plasma enthalpies for dc arc torches are shown in Fig. (8).

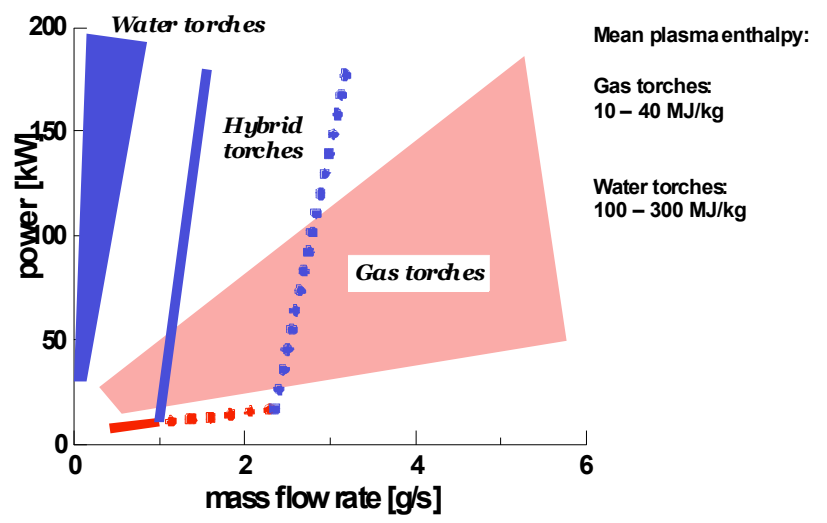

Fig. (8). Operation regimes of $\mathrm{dc}$ arc plasma torches.

As plasma mass flow rate in Gerdien arc is controlled by arc processes, it can not be controlled independently of arc power. To cover the region of parameters between gas and water torches and to control plasma mass flow rate, a new type of plasma torch, based on combination of principles of arc stabilization by gas flow and water vortex, was designed. The torch is described in the following paragraph.

\section{HYBRID WATER/GAS STABILIZED PLASMA TORCH}

Physical limits of the two principles, the gas and the liquid arc stabilization, do not allow generation of plasmas with parameters in a wide range between the two principles. Therefore new type of plasma torch, hybrid gas/water torch, was designed [19]. In this torch principles of arc stabilization by the gas flow and by the water vortex are combined.

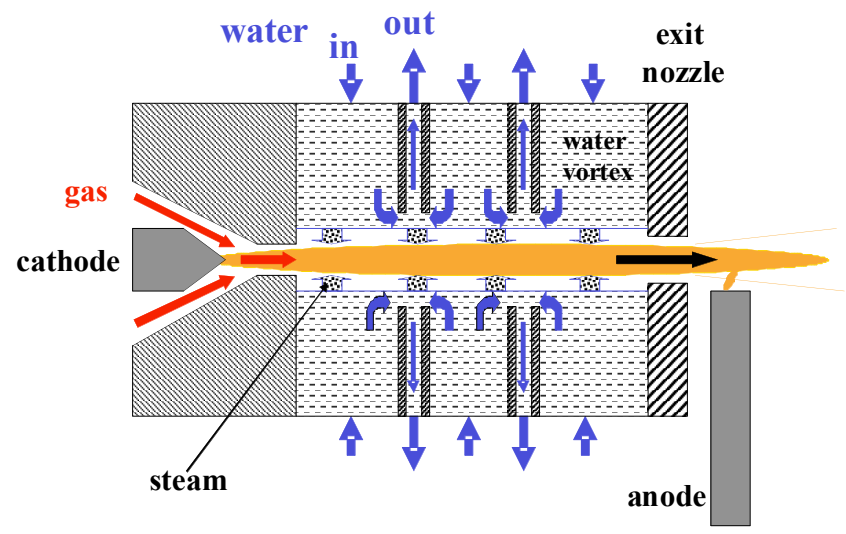

Fig. (9). Schematics of hybrid gas/water torch.

The schematic diagram of hybrid plasma torch is shown in Fig. (9). The cathode part of the torch is arranged similarly like in gas torches. Gas is supplied along tungsten cathode tip, vortex component of gas flow that is injected tangentially assures proper stabilization of arc in the cathode nozzle. Gas plasma flows through the nozzle to the second part of arc chamber where arc column is surrounded by a vortex of water. The vortex is formed in a chamber with tangential water injection in a same way like in waterstabilized torch. The sections with tangential water injection are separated by two exhaust gaps, where water is exhausted out of the arc chamber. Interaction of the arc column with the water vortex causes evaporation from the inner surface of the vortex. The steam mixes with the plasma flowing from the cathode section. An anode is created by a rotating copper disc with internal water cooling like in water torches. The arc column is composed of three sections. The cathode section is stabilized by a vortex gas flow. If gas with low enthalpy like argon is used, the voltage drop and power of this section is small. The most important section is the water-stabilized part, where the arc column interacts with the water vortex. The third part between the exit nozzle and the anode attachment is an arc column in a free jet formed from mixture of argon with steam. In experimental torch the length of the gas-stabilized arc column was about $6 \mathrm{~mm}$, the length of the water-stabilized column was $50 \mathrm{~mm}$, the length of arc in free jet between exit nozzle and anode attachment varied in the range of 4 to $24 \mathrm{~mm}$ as the anode attachment moved along the electrode surface in the restrike mode.

The way how operation regime is established in a hybrid torch is illustrated in Fig. (8). In the cathode gas-stabilized section the power increases with gas flow rate slowly, if low enthalpy gas like argon is used (red part of characteristics in Fig. (8)). Energy balance in the water stabilized arc section is almost completely controlled by steam inflow and the arc in this section has electrical characteristics and power balances that are very close to the ones of water-stabilized torches. The power thus increases rapidly with mass flow rate as in the case of water torch (blue part of characteristics in Fig. (8)).

The arc voltage and thus arc power are almost completely controlled by the water stabilized part. In Fig. (10) power balances of hybrid plasma torch are shown. The power 
dissipated in cathode gas stabilized part for arc currents range of $200 \mathrm{~A}$ to $450 \mathrm{~A}$ was only $2 \mathrm{~kW}$ to $6 \mathrm{~kW}$ [19]. Great differences in power dissipated in gas stabilized and water stabilized sections result from differences in the length of corresponding arc columns and from the fact that enthalpy of steam plasma is substantially higher than enthalpy of argon plasma. Plasma temperature and characteristics controlled by energy balance are thus close to values typical for water torches, while mass flow rate can be controlled by changing flow rate of argon.

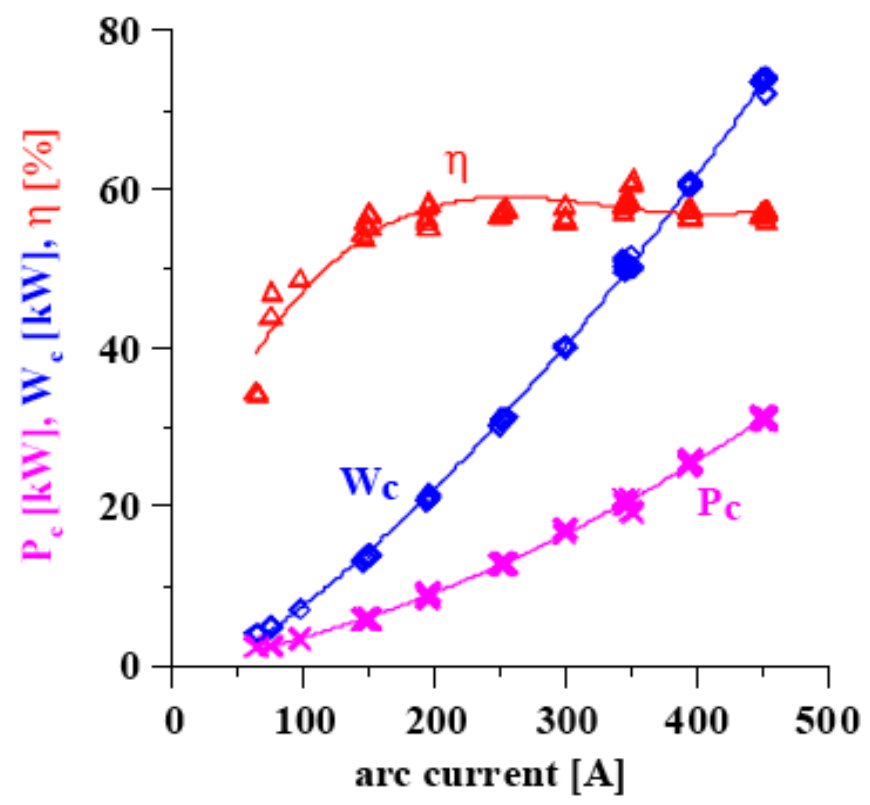

Fig. (10). Power dissipated in the part of arc column stabilized by water vortex $W_{c}$, power loss to water $P_{c}$ and efficiency $\eta$ in dependence on arc current for hybrid argon/water torch. Argon flow rate $30 \mathrm{slm}$.

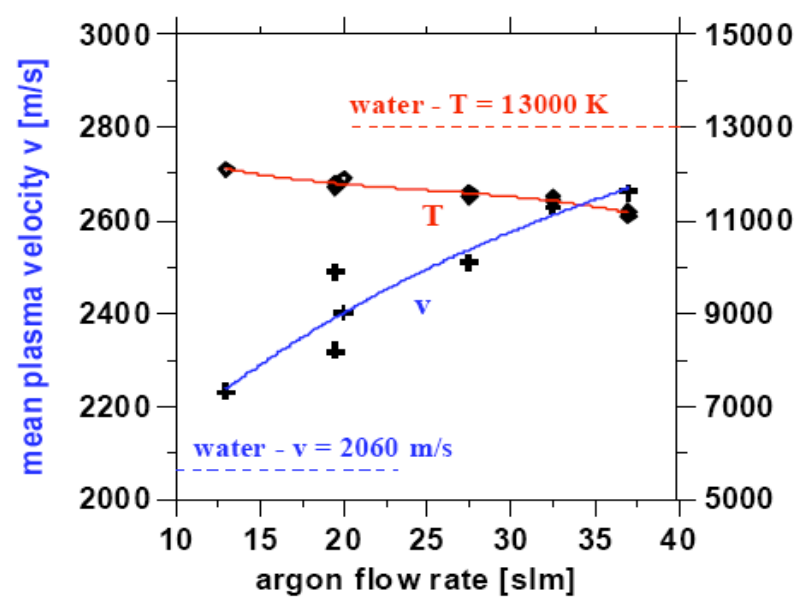

Fig. (11). Mean plasma temperature and velocity at the torch exit in dependence on argon flow rate. $\mathrm{I}=350 \mathrm{~A}, \mathrm{P}=100 \mathrm{~kW}$. Values measured in pure water torch with the same power are indicated by broken lines.

Characteristics of the torch and properties of plasma jet were measured for arc currents of 200 - $500 \mathrm{~A}$, arc powers of $30-130 \mathrm{~kW}$, and argon flow rates of $8-28 \mathrm{slm}$ [19]. The volume percentage of argon in the plasma flow at the torch exit depends not only on the argon flow rate, but also on the arc current because the evaporation rate of the stabilizing water increases with an input power. The volume percentage of argon varied approximately from 20 to $80 \%$. The centreline plasma flow velocity at the torch exit, increasing with both current and argon flow rate, ranges approximately from $1800 \mathrm{~m} / \mathrm{s}$ to $7000 \mathrm{~m} / \mathrm{s}$. The centerline exit temperature is almost independent of argon flow rate and varies between 14 $\mathrm{kK}$ and $22 \mathrm{kK}$. The plasma temperatures were somewhat lower than the ones measured in water-stabilized torches, the plasma velocity was significantly higher. Dependence of mean plasma temperature and velocity on argon flow rate is shown in Fig. (11). For comparison, values measured for pure water torch at the same arc power are shown in the figure.

\section{CONCLUSIONS}

Plasma torches with Gerdien (water-stabilized) arc are used in industrial scale for plasma spraying, other plasma chemistry applications like waste treatment or gasification of biomass for syngas production are being investigated. Oxygen/hydrogen plasma is produced in Gerdien arc by heating and ionization of steam that is evaporated from water vortex which confines the arc column. This principle of stabilization of arc offers possibility of generation of plasma jets with extreme plasma enthalpy and very low density. A new type of arc with combined stabilization by gas flow and water vortex has been investigated recently.

Water torches are characterized by high arc power and low mass flow rates and especially very low ratio of mass flow rate to the arc length. This leads to extremely high plasma enthalpy and temperature. Thermodynamic and transport properties of steam plasma lead to high heat transfer to the injected material by thermal conduction. High ratio of plasma enthalpy to electrical conductivity results in high arc voltage and thus high arc power. The principal process controlling the arc properties is radial transport of energy dissipated in the conducting arc core by Joule heating. The relations between the power absorbed in steam sheath surrounding the arc column, the power absorbed in the boiling layer on the inner surface of water vortex and the power transferred into the water body are decisive for all arc and plasma parameters. Especially the ratio of the power spent on water vaporization, i.e. on the production of plasma gas, to the power absorbed in the produced vapor and spent for its heating, is a critical factor. Evaluated fraction of the total power consumed for water vaporization was approximately 0.01 while for steam heating about 0.6 of total power was spent. This is the principal cause of high plasma temperatures and velocities found in the experiments.

Substantial increase of range of plasma jet parameters can be achieved in hybrid torches with combined gas-water stabilization. If low enthalpy gas like argon is used, energy balance in the arc column is almost completely controlled by steam inflow and the arc has electrical characteristics and power balances that are close to the ones of water-stabilized torches. On the other hand mass flow rate and momentum flux are strongly influenced by gas flow and can be thus controlled almost independently of power balance. In plasma spraying the hybrid torches provide thermal efficiency almost the same as water torches but particle velocity can be increased substantially.

Extremely high plasma enthalpy and plasma temperature and very low plasma density result in very high efficiency of 
utilization of plasma enthalpy for heating of material injected into plasma. This is advantageous in all applications where high heat fluxes and high temperatures are needed. Especially high throughputs of treated material and high process rates are characteristic for water plasma torches. In plasma spraying the water-stabilized torches provide almost one order higher spraying rates than gas torches. Therefore water-stabilized systems are used for large-area coatings, for production of self-supporting ceramic parts and for powder processing [20]. Several promising applications of oxygenhydrogen plasma jets appear besides plasma spraying, namely waste treatment and plasma gasification of biomass for syngas production [21]. Besides high level of turbulence, high rate of mixing process and high plasma enthalpy also chemical composition of plasma is advantageous for this application.

The author would like to thank to the Grant Agency of the Czech Republic for support of this work under the projects No. 202/08/1084.

\section{REFERENCES}

[1] Fauchais P, Vardelle A. Thermal plasmas. IEEE Trans Plasma Sci 1997; 25: 1258-80.

[2] Heberlein J, Murphy AB. Thermal plasma waste treatment. J Phys D Appl Phys 2008; 41: 1-20.

[3] Gerdien H, Lotz A. Wasserstabilisierter Lichtbogen. Wiss Veroff Siemenswerk 1922; 2: 489-92.

[4] Gerdien H, Lotz A. Uber eine lichtquelle von Sehr hoher flachenhelligkeit Z Tech Phys 1923; 4: 157-60.

[5] Maecker H. Ein Lichtbogen für höhe Leistungen. Z Phys 1951; 129: 108-22.

[6] Burnhorn F, Maecker H. Feldstärkemessungen an wasserstabilisierten Hochleistungsbögen. Z f Phys 1951; 129: 369-76.

[7] Larentz RW. Temperaturmessungen in der Säule eines GerdienBogens. Z Phys 1951; 129: 343-64.

[8] Burnhorn F, Maecker H, Peters T. Temperaturmessungen am wasserstabilisierten Hochleistungsbogen. Z Phys 1951; 131: 28-40.
[9] Gross B, Grycz B, Miklossy K. Plasma technology. London: Iliffe Books Ltd 1968.

[10] Hrabovsky M, Kopecky V, Sember V. Water stabilized arc as a source of thermal plasma, In: Fauchais P, Ed. Heat and Mass Transfer under Plasma Conditions, New York - Wallingford: Begell House 1995; pp. 91-8.

[11] Hrabovsky M, Konrad M, Kopecky V, Sember V. Processes and properties of electric arc stabilized by water vortex. IEEE Trans Plasma Sci 1997; 25: 833-9.

[12] Hrabovsky M. Water-stabilized plasma generators. Pure Appl Chem 1998; 70: 1157-62.

[13] Hrabovsky M, Konrad M, Kopecky V, Sember V. Properties of electric arc stabilized by mixture of water with ethanol. Ann N Y Acad Sci 1999; 891: 57-63.

[14] Jenista J. Water-vortex stabilized electric arc: I. numerical model. J Phys D Appl Phys 1999; 32: 2763-76.

[15] Jenista J. Water-vortex stabilized electric arc: II. effect of nonuniform ervaporation of water. J Phys D Appl Phys 1999; 32: 2777-84.

[16] Hrabovsky M, Krenek P. Properties of thermal plasma generated by the torch with water stabilized arc, in $11^{\text {th }}$ International Symposium on Plasma Chemistry Loughborough, Conference Proceedings. Harry J, Ed 1993; vol. 1: pp. 315-20.

[17] Krenek P, Hrabovsky M. $\mathrm{H}_{2} \mathrm{O}-\mathrm{Ar}$ plasma property functions for modeling of hybrid water - gas plasma torch, in $18^{\text {th }}$ Int Symp on Plasma Chemistry, Kyoto. Conference Proceedings. Tachibana K, Takai O, Ono K, Shirafuji T, Eds. Book of Abstracts, 75, full paper on CD 2007.

[18] Hrabovsky M, Konrad M, Kopecky V. Integral model of arc column stabilized by liquid vortex, in XIII International Conference on Gas Discharges and their Applications. Glasgow, Conference Proceedings. Scott J, MacGregor J, Eds. 2000; pp. 11821.

[19] Hrabovsky M, Kopecky V, Sember V, Kavka T, Chumak O, Konrad M. Properties of hybrid water/gas dc arc plasma torch. IEEE Trans Plasma Sci 2006; 34: 1566-75.

[20] Chraska P, Hrabovsky M. An overview of water stabilized plasma guns and their applications, in International Thermal Spray Conference. Orlando, Conference Proceedings. Berndt C, Ed. Florida, 1992; pp. 81-5.

[21] Hrabovsky M, Konrad M, Kopecky V, Hlina M. Pyrolysis of wood in arc plasma for syngas production. J High Temp Mat Process 2006; 10: 557-70. 